\title{
Advances in Nano-Bio-Optics: Detection from virus towards higher sized biostructures
}

\author{
Amé $\mathrm{M}^{1}$ and Bracamonte $\mathrm{AG}^{2,3 *}$ \\ ${ }^{1}$ Hospital Privado e Instituto Universitario de Ciencias Biomédicas de Córdoba, Córdoba, Argentina \\ ${ }^{2}$ Instituto de Investigaciones en Físico Química de Córdoba (INFIQC), Departamento de Química Orgánica, Facultad de Ciencias Químicas, Universidad Nacional \\ de Córdoba (UNC). Ciudad Universitaria, 5000 Córdoba, Argentina \\ ${ }^{3}$ Departement de chimie and Centre d'optique, photonique et laser (COPL), Université Laval, Québec (QC), Canada, G1V 0A6
}

\begin{abstract}
In this communication were discussed Nano-Optics developments for targeted Biodetection applications. In this context, it was presented the last publications related with Antibodies-antigen interactions involucrated for Corona Virus (COVID-19) detection, as well as other approaches of non-covalent interactions for higher sized Biostructure detection based on Bioimaging generation and Optical signaling modifications. In this context it was presented the development of Lab.-on-particles and Functional Nanoparticles tuned for Biodetection. By this manner, it was discussed the potential use of targeted Nanoplatforms for light delivery applications. In this way, it was showed the importance of the control of the Nanoscale with tunable Optical properties for variable sizes of Biostructures. Moreover, in this particular topic it was introduced in Advance the theme related with the generation of Nanolasers, Biolasers and Living Lasers with potential uses within Biosciences, such as Biodetection and new Nano-Photo-thermal treatments.
\end{abstract}

Thus, it was afforded to introduce different insides within from the control of Nano-Optics towards Nano-Bio-Optics for detection of different Biostructure sizes with the potential capability to detect internal Biomolecules and Biological processes.

So, different themes and topics were discussed in order to stimulate Research and Development within Nano-Optics and Nano-Biophotonics for Biodetection applications. In addition it was contemplated as well developments within Nanomedicine.

\section{Introduction to bio detection}

The Biodetection is a particular field with non-controlled variables conducted by the intrinsic matter nature and Bio-components involucrated in complex real matrixes. So, it should be contemplated variations due to interferences from the media too. Thus, the development of Nano-Optical platforms [1-3] could afford to target and detect different types of Biostructures. It is need it the development of new Biodetection methodologies for Virus [4] Bacteria [5] Unicellular Micro-organisms [6] and cells [7] related with different illness.

In order to develop targeted Biodetection methodologies should be considered the main variables such as i) sizes of Biostructures ii) membrane constitution iii) internal complexity iv) size of Nanoplatforms v) chemical surface modification of Nanoplatforms vi) tuning of Nano-Optical properties vii) others etc. Moreover, it should be taken into account the Biological variability. This variable mentioned could be easily exemplified with the current Pandemic state related with the severe acute respiratory syndrome coronavirus 2 (SARS CoV-2) [8] generated by a small Virus in the Nanoscale with variable genetic information and Biomembrane constitution [9] that could affect in different manners depending of the genetic of the host [10] as well.

In similar manner, if it considered Bacteria it should be contemplated a large variability of Biostructures associated at different illness. For example, it could be mentioned the well-known Escherichia coli with Clinical implications [11] and Cyanobacteria as Unicellular microorganisms [12]. In this subject it should be mentioned the variable sizes considered from the higher Nano-scale to the Micrometer scale.
In this direction, in the present communication was presented recent advances of Biodetection by the use of the Nano-Optical platforms joined to Bioconjugation strategies.

The control of the nanoscale for virus, bacteria and unicellular organism detection

The control of the Nanoscale could involucrate different implications such as Biostructure trapping within porous Polymeric materials $[13,14]$ and by Optical Tweezers $[15,16]$ Biostructure depositions on Lab On-particles [17] and Nano-Biolabelling [18] applications. Thus, the design and synthesis of Nano-Micro-platforms within Colloidal dispersions as well as Nano-, and Micro-patterned surfaces are of high impact within Life Sciences.

In this way, it should be known that Virus showed sizes within the Nanoscales such as for Corona Virus with 100-120 nm [19] and Zika Virus with $50 \mathrm{~nm}$ cite [20]. While, bacteria could variate sizes from

${ }^{\star}$ Correspondence to: A. Guillermo Bracamonte, Instituto de Investigaciones en Físico Química de Córdoba (INFIQC), Departamento de Química Orgánica Facultad de Ciencias Químicas, Universidad Nacional de Córdoba (UNC). Ciudad Universitaria, 5000 Córdoba, Argentina, E-mail: gbracamonte@fcq.unc. edu.ar, guillermobrac@yahoo.ca

Key words: Biodetection, Virus, Bacteria, unicellular microorganisms, NanoBiophotonics, Nano-Bio-Optics, Nano-Optics

Received: April 14, 2020; Accepted: April 22, 2020; Published: April 29, 2020 
the higher sized Nano-scale towards micrometers [21], and Unicellular microorganisms, cells in higher sized micrometer scale [22]. Moreover, it should be noted that targeted membrane constituents could variate from small molecules below and close of the Nanoscale (around 1 $\mathrm{nm}$ ); and peptides, proteins, Glico-proteins and antibodies in smaller intervals of sizes of the Nanoscale. In this context, recently it could be mentioned the targeted distinct conformational states of SARSCoV-2 spike protein [23] and their use for targeted Biodetection and treatments based on Immuno-approaches. For example, the isolation of potent SARS-CoV-2 neutralizing antibodies showed protective effect from disease in a small animal model [24]. In this way, looking for improvements it was an antibody cocktail of SARS-CoV-2 spike protein that prevented rapid mutational escape seen with individual antibodies [25]. Thus, this showed a possible opened solution to the extra difficulty in particular of this illness related with its fast genomic mutation. In similar manner, it was recorded a large and broad neutralization monoclonal antibodies of SARS-related viruses [26] In addition, it should be added the role of nanotechnology in the treatment of viral infections [27] by the developments of Nanoparticle, Nano-carriers, Nanoaggregates that could interact at different levels in the Virus structure [28] (Figure 1), mechanism of action, and pathway within the host [29]. For example, by interfering the interaction, adsorption, internalization, RNA replication, etc.

In particular, in this communication it is highlighted the importance and implication of the tuning and use of varied Nanoparticles and Nanoplatforms to interact and provide by different strategies new detection approaches and treatments. In this manner, it could be mentioned the Nanostructures for the Inhibition of Viral Infections [30] based in variable material compositions and properties associated. For example the study of interaction of silver nanoparticles with Tacaribe virus [31] for potential antibiotic action, and Carbon based Nanodots with high Efficiency as entry Inhibitors of Herpes Simplex Virus Type 1 [32].

Moreover, it should be mentioned the broad-spectrum of non-toxic antiviral nanoparticles with a virucidal inhibition mechanism [33]. This mechanism consisted in the prevention of the first step of virus-cell interaction by mimicking heparan sulfate proteoglycans (HSPG) linked to Nanoparticles, to target viral attachment ligands (VALs). Thus, it was effectively attached on the Viral sites that generated irreversible shape deformations and effect modification. Other example, the virucidal activity of reduced sized Gold Nanoparticles synthesized by Green Chemistry using Garlic Extract [34], via a mechanism of deposition on

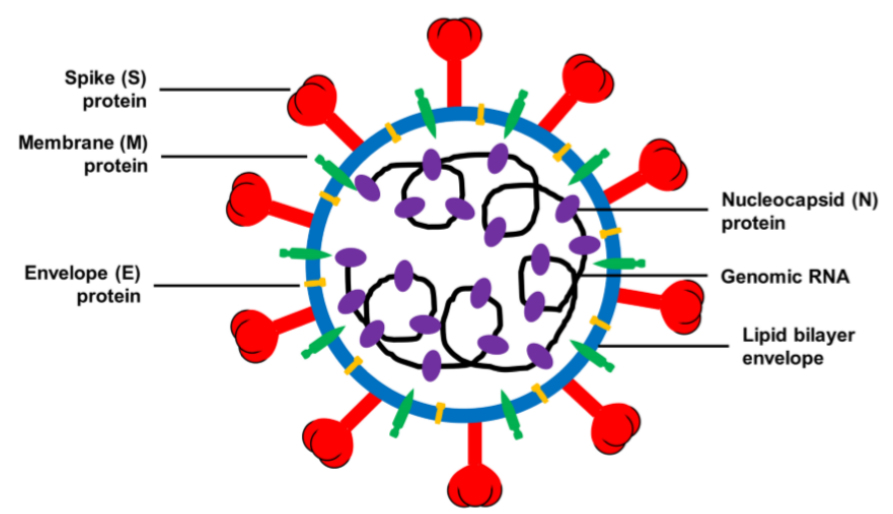

Figure 1. Schematic structure of virion of SARS-CoV, MERS-CoV, and 2019-nCoV and its major structural proteins. Reprinted with permissions of L. Du et al. (cite 28), Front. Microbiol, Frontiers 2020 the active binding sites and deactivation of the Virus. To these properties of gold Nanoparticles related with Biocompatible properties combined with tuneable surfaces, sizes, with electro-active properties; it should be mentioned the Photo-thermal properties by Laser excitation, and the generation of high reactive species such as radicals [35].

In the previous mentioned examples were showed how the noncovalent interaction of different Nanostructures could acted by variable mechanism such as the followed strategies: i) simple contact of intrinsic potential antibiotic action properties from silver, ii) combination of hydrophobic properties of Carbon dots as entry inhibitors agents, iii) Biomolecular targeted membrane surfaces with a consequent membrane deformation, and iv) reduced sized gold Nano-blocking agents, accompanied with potential properties as, iv) intrinsic Optical and Electronic properties from gold Nano-surfaces related with High Energy Electromagnetic fields.

In addition, within higher sized Biostructures could be mentioned similar examples such silver nanoparticles as antimicrobial agent: a case study on E. coli as a model for Gram-negative bacteria [36]. However, due to the different ways of actions, sizes involucrated, and types of Biostructures, it could be expanded to other strategies. In the next, section it was discussed how from the control of synthetic Nanoarchitectures it could be tuned Optical properties from single Nanoplatforms for Multi-functional Bio-applications.

\section{Nano-optics and nano-bio-optics from viruses towards higher sized biostructures}

The developments of controlled Nano-Optical platforms by the right selection and tuning of Optical active Nanomaterials [37] leads with Multidisciplinary fields Research studies. In this manner could be generated variable smart responses against external stimulus that affects their intrinsic Optical properties. These mentioned properties are related with their electronic Nanomaterials properties and their interaction by Light excitation. Thus, Photonics and Nanophotonics showed high impact in other different Research fields; where Chemistry, Physics and Clinical Spectroscopy were closely involucrated.

There are a many Optical active properties that could be developed to tune on Optical-Nanoplatforms; however in particular in this communications it was discussed the use of: i) Optoelectronic phenomena such as the generation Electromagnetic fields within the near fields of metallic Nanoparticles; ii) Plasmonic properties coupled to other Light pathways, iii) generation of non-classical Light phenomena, and iv) Meta-Photonics. These Research themes were discussed in the context of detection of Virus and higher sized Nano, and Micro-Biostructures by the use of different of varied Optical detection approaches and strategies.

In the same way of the development of the Nano-Optical platforms for Biodetection, it should be contemplated different strategies for targeting particular Biostructures. Thus, Bioconjugation with Biomolecules, antibodies, and synthetic chemical structures should be contemplated depending of the membrane composition and media related. So, the design and synthesis of modified Nano-Optical platforms could generate Multifunctional Nanoparticles as Lab.-Onparticles [38] where varied functionalities and targeted applications are ready to be activated by external controlled response od via Smart responsive strategies.

In this manner, as example of Advance Nano-Optical platforms for an accurate control of High Energy Electromagnetic Fields by an Optical guiding, it could be mentioned the reversible shape and 
Plasmon Tuning in Hollow Ag/Au Nanorods [39]. The particular Ag/ $\mathrm{Au}$ Nanorod structures were generated by partial galvanic replacement and manipulated their internal cavity reversible by Laser excitation. This strategy permitted to control their Electromagnetic fields known as Plasmons with a nanometer resolution (Figure 2). The changes in plasmon-induced near-field properties were then mapped with electron energy-loss spectroscopy without disturbing the internal structure. This reversible shape and near-field control in a hollow nanoparticle actuated by an external stimulus introduces possibilities for applications in reprogrammable sensors, responsive materials, and optical memory units.

Moreover, the generation of non-classical light emission and control with an accurate Nano-resolution, it could be tuned and developed methodologies for Bio-molecules detections towards Biological structures if it is targeted these applications. In this context, the previous example with strong Electromagnetic fields generation could interact with their close surrounding to sense its environmental changes that it could be related with different targeted Biostructures of interest.

The Metal Enhanced Fluorescence (MEF) is phenomena related with the enhanced excitation of Fluorescent reporters within shorter Nano-scale interval of lengths $[40,41]$. By this manner, it was developed by us the tuning of Ultraluminiscent gold core-shell Nanoparticles as NanoImaging Platforms for Biosensing applications based on Metal enhanced fluorescence (MEF) [42] with MEF Enhancement Factors $\left(\mathrm{MEF}_{\mathrm{FF}}\right)$ that arrived to 40 with Laser Fluorescence Microscopy. These types of Nano-emitters showed Ultraluminescent properties accompanied with diminished photobleaching properties. The implication of the control of light from the Nanoscale has impact on the Nano-resolution, with applications such as super-resolution Nanoscopy by coherent control on nanoparticle emission [43]. In addition, these properties and resolutions could be translated on Biostructures, or smaller sized Biostructures deposed on Nano-, and Microplatforms. At the same time, these Higher Electromagnetic fields and emissions could generate High Photo-thermal properties with potential uses in for developments of new Photo-thermal treatments [44]. As example, it could be mentioned as recent communication the photothermally enhanced tumor vascular disruption treatment [45] induced by the

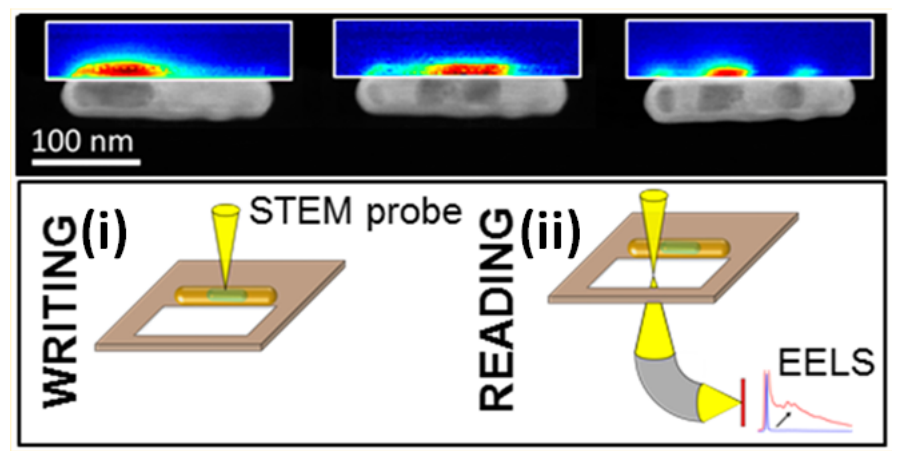

Figure 2. Electron Energy-Loss Spectra (EELS) spectra and maps of the three void positions. Plasmon resonances and the relationship between energy electron-loss probability and internal structure in a partially hollow $\mathrm{AgAu}$ nanorod for the corner state before any reshaping cycle, center state, and split state; for then corner state after a reshaping cycle. Insert image. Write/read cycle and reversibility of interior restructuring in partially hollow AgAu nanorods: i) Ag migrates toward the beam when a solution-filled volume is illuminated, ii) The void position and its effect on plasmon resonances can be interrogated without restructuring by acquiring electron energy-loss spectra (EELS) from the surroundings of the nanorod. Reprinted with permissions of D. Boudreau-E. Ringe et al. (cite 39), Nano Lett. ACS 2016 aggregation of gold nanoparticles deposed on targeted modified vascular tissues.

So, in the context of the Pandemic situation it should be highlighted the importance to mention the actual methodologies applied for Corona Virus detection as well as other Biostructures such as Bacteria and other Micro-organisms. In this way, it could be mentioned that the Biodetection of Viruses nominated as Cargo Biostructures with genetic information could be sequenced and amplified by several sequencing technologies such Polymerase Chain Reaction (PCR) [46] and new developments of methodologies related [47] currently in progress. This technique could contemplate the problem associated with the genetic variability [48] due to its precision. Thus, it was tracked the positive rate of RT-PCR detection of SARS-CoV-2 infection in 4880 cases from one hospital in Wuhan, China, from Jan to Feb 2020 [49].

Moreover, it should be highlighted that actually the bacteria detection with Clinical implications such as Escherichia coli in order to confirm their presence and concentrations it is used Bright Field Confocal Microscopy with glass chambers for their counting [50]. However, it is need faster and more accurate methodologies with portable instrumentation to detect in different places with the possibility of keep moving on demands. In this way, in the current times it was showed the capability to response by a rapid implementation of mobile technology for real-time epidemiology of COVID-19 [51] But, it is not all developed, so the Research from basic studies towards applied Nanotechnology as tools or being part of Instrumentation could be still being developed. For this reason the Nanospectroscopy could afford to different developments in the recent mentioned needs. In this direction, it could be mentioned the use of Spectroscopy for Clinical studies and analysis such as Translation of diagnostic IR, 2D-IR and Raman spectroscopy towards the clinic uses [52] and related with Biostructures the diagnosis of hepatitis $\mathrm{B}$ and $\mathrm{C}$ virus infection from human sera using ATR-FTIR spectroscopy [53].

About, Nanoarchitectures with different Optical properties for targeted Viruses detection accompanied with their respective treatments, it could be mentioned recent publications such as Functional Carbon Quantum Dots as Medical Countermeasures to Human Coronavirus [54] and polydopamine doped virus like structured nanoparticles for photoacoustic imaging guided synergistic chemo-/Photothermal therapy [55]. The use of Optical Detection of Viruses by the use of metallic Nanoparticles was already used for others viruses [56] such as for the HIV infection with streptavidin conjugated gold nanoclusters. This strategy, based on a Fluorescent Nanosensor, showed to be an ultrasensitive early diagnosis tool for Biodetection of HIV [57]. Then, other important contribution of the control of the Nanoscale was by generation of porous Nanomaterials as cellular Nano-sponges to inhibit the SARS-CoV-2 Infectivity [58].

The different approaches showed different properties that could be placed within modified substrates being part of reduced sized devices or miniaturized portable Instrumentation as for example developments on Chips with Optical detections [59].

In this direction as well it could be highlighted as well the recent development of Thermoplasmonics SARS CoV-2 detection system based on Surface Enhanced Raman Scattering (Figure 3) [60]. In this Research work, it was combined a Plasmonic Photothermal (PPT) with Localized Surface Plasmon Resonance (LSPR) sensing. The PPT treatment generated the free genomic material to be targeted by functionalized gold Nano-islands with complementary DNA strands. Thus, it was achieved a high sensitive detection approach for SARS 
CoV-2 through in situ hybridization and detection via Plasmon Laser excitation. By this manner, it was arrived to a lower detection limit below $0.22 \mathrm{pM}$.

However, it should be highlighted the genotyping based on Optical active Nanoplatforms, by Single Optical-active Nanoparticle analysis with imaging within an In Flow Optical set up. By this manner, it was showed low values such femto-M concentration for similar lengths of single DNA strands in comparison to the SARS CoV-2 DNA lengths [61].

The detection it was developed based on a coupled Plasmonic methodology by Fluorescence Resonance Energy Transfer (FRET) and MEF previously discussed [62]. This Nano-Biosensor, it was validated in blood samples as PCR free genotyping applications such as it was validated for the Sex Determining region known as SRY [63].

In many of the previous examples it was showed how OpticalNanoplatforms based on their intrinsic matter constitution and tuning generated High Electromagnetic fields coupled to other phenomena to improve and Enhance sensitivities, detection and Energy transfer. Moreover, these Optical properties were as strong enough to be applied for Optical tweezers Set-ups. This technique showed high sensitivity and sensitivity to control molecules, Nano-, and Microparticles and even Biostructures. For example, it could be mentioned the Nanooptical Trapping of Rayleigh Particles and Escherichia coli Bacteria with Resonant Optical Antennas (Figure 4) [64].

The patterned metallic nanoantennas on a glass substrates produced strong light intensity gradients responsible for the trapping mechanism of several individual bacteria simultaneously with their orientation fixed by the asymmetry of the antennas.

In similar manner considering the Biodetection of higher sized Biostructures such as Escherichia coli bacteria, but using a approach based on Ultraluminescent Nanolabellers, it was developed a NanoBiolabelling methodology that permitted well defined Eschericcia coli bacteria imaging by Laser Fluorescence Microscopy. In this manner it was achieved individual bacterial detection based on MEF NanoBioimaging (Figure 5) [65].

The Core-shell Nanoparticles bellow $100 \mathrm{~nm}$ sizes previously mentioned were homogeneous deposed on membranes of 100-1500 nm longitudinal lengths of Escherichia coli based on polar non-covalent interactions between Biomolecules and Silanol groups of the Silanized Nano-surfaces. These Nano-Biostructures showed stable Dynamics within Colloidal dispersions that permitted the development of an In

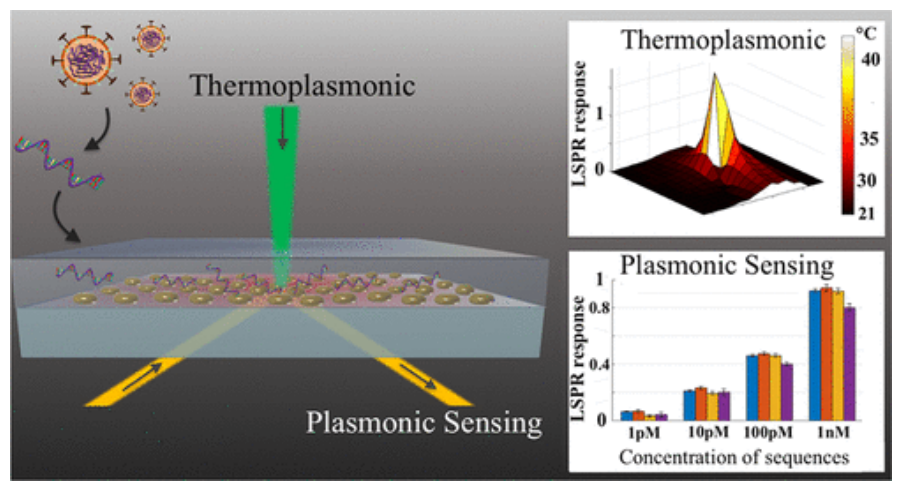

Figure 3. Schema of Thermoplasmonics approach based on Surface Enhanced Raman Spectroscopy (SERS). Reprinted with permissions of J. Wang et al. (cite 60), ACS NANO, ACS 2020
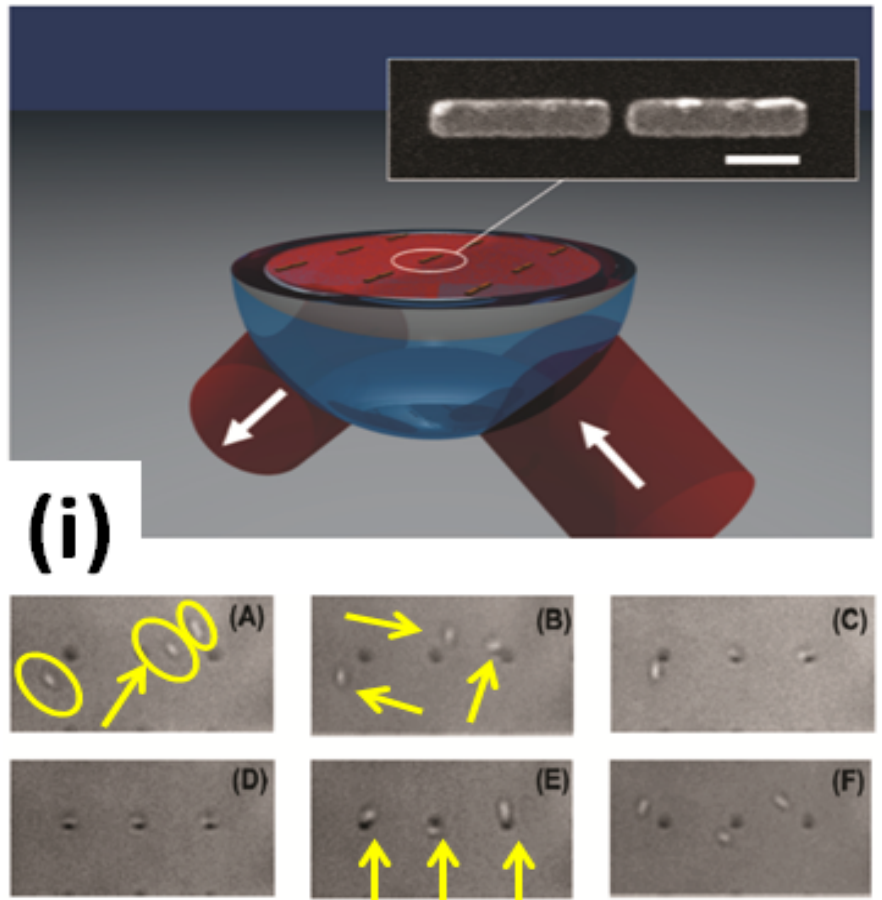

Figure 4. Periodically arranged gold optical nanoantennas, lying on a glass substrate and immersed in water, are illuminated under total internal reflection through a hemispherical glass hemispherical lens. Inset: SEM micrograph of a single gold gap antenna (scale bar: $200 \mathrm{~nm}$ ). Inset image i): (A-F) Successive frames showing simultaneous trapping of E. coli bacteria. The incident laser $(800 \mathrm{~nm})$ has been switched off just before recording frame E. Reprinted with permissions R. Quidant et al. (cite 64), Nano Lett., ACS 2009
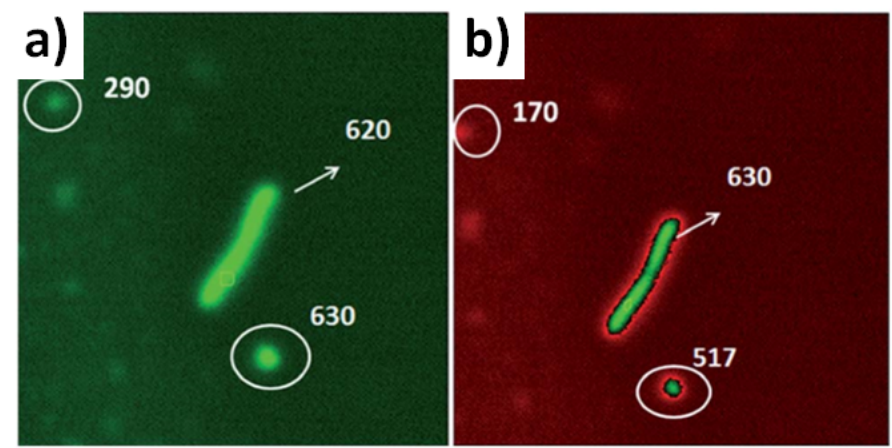

Figure 5. Laser fluorescence microscopy images of ultraluminescent bacteria labelled with $\mathrm{Au} @ \mathrm{SiO} 2-\mathrm{RhB}$, silica spacer $14 \mathrm{~nm}$ : a) edited by green colour; and b) edited by red, green colour. Reprinted with permissions of D. Boudreau-A. G. Bracamonte et al. (cite 65), J. of Nanophotonics, SPIE 2018

Flow MEF-Bioassay for Biodetection of Escherichia coli [66]. By this manner, it was showed the versatility of the design of Nano-Optical platforms for varied uses related with Biodetection. However, it should be noted that there is a huge opened window and different needs to overcome that affords to new Nanotechnology developments towards Nano-Biotechnology.

Finally, it is highlighted another recent publication as well with the concept of non-classical Light delivery applications within Optical active higher sized Uni-cellular micro-organisms such as Cyanobacteria. In this approach it was optimized the synthetic non-classical luminescence generation by Enhanced Silica Nanophotonics based on Nano-BioFRET [67] (Nano-Bio-Fluorescence Resonance Energy Transfer), that permitted not only to detect this Unicellular micro-organism, but as well it was sensed and discriminated both natural Photo-systems 
incorporated within these Biostructures. This Biosensing was by controlling the Laser excitation and emission that generated strong emissions coupling depending of the excitation applied. These NanoBiostructures are related as well with the generation of Living Lasers [68] and Biolasers [69]. Thus, these themes overloaded the extension of this section, but it is merited to be mentioned for a next opportunity still discussing about these phenomena within Life Sciences joined to Nanotechnology.

\section{Concluding remarks}

It was discussed the design of Optical Nanoplatforms for Biodetection applications of different sizes of Biostructures and their future perspectives by joining new treatments. The Optical properties discussed on Nano-, and Microparticles were based on different tuneable intrinsic matter properties by chemical, physical and Laser methodologies. It was of our interest to show some results in progress recently published by us in the Research field of NanoOptics developments of High Energy Electromagnetic fields generation from metallic Nano-surfaces, in order to be used as Nanoplatforms for Bioconjugation.

In this way, it was afforded the Biodetection from Virus towards higher sized Biostructures such as bacteria, unicellular microorganisms and Cells. Thus, it was considered the detection based on modified surfaces and Lab-On particles; where non-covalent interactions were involucrated coupled to non-classical light pathways for detection. By this manner, antibodies antigens interactions were discussed as for example for accurate targeting of SARS CoV-2 detection.

In this manner, it was intended to discuss and motivate about the potential role of Biotechnology developments based on Nanophotonic developments; where it could be combined improved detection, systems coupled to future new treatments. For example, for the COVID 19 detection it should be contemplated from the interactions between the spiked protein of SARS CoV-2 with the cell receptors towards the internalization within the cells with the consequent proliferation of the Virus, to develop targeted combinatory treatments [70]. Therefore, it should be known the different chemical environment where the interactions are involucrated as well as their local chemical environment, such as varied amino acid compositions of antibody proteins. This mention is related with mutations that could provide variable hydrophobic aliphatic regions, neutral aromatic moieties, thiol and sulfides groups, carboxylic and hydroxyl groups with hydrophilic properties, etc. by modifying their genetic expression. So, the Biological variation factor added to the Biodetection was showed to be an extra challenge to overcome. Moreover, after internalization of the Biostructure it should be contemplated extra interactions such replicating RNA for joining therapies and vaccines. However during the while, in the context of Pandemic situations it should be highlighted the prevention factor by isolation and physical distancing to avoid the dissemination of the SARS-CoV-2, as it was recently reported the shortening over time by non-pharmaceutical interventions [71].

In addition, it was considered the developments of Nano-Optical platforms for higher sized Biostructures detection based on different strategies and phenomena. By this manner, it was showed examples of bacteria such Escherichia coli; and Uni-cellular microorganism as Cyanobacteria.

So, different aspects were discussed, such as from the design of Nano-Optics, towards interactions with Bio-membranes for targeted Biodetection of different types of Biostructures.

\section{Acknowledgment}

We would like to gratefully acknowledge the different Grants received in order to accomplish the Research work in progress of the authors related with developments of Luminescent Nanoplatforms and Biodetection, "CONICET, Consejo Nacional de Investigaciones Científicas y Técnicas (National Research Council of Argentine); ANPCyT, Agencia Nacional de Promoción Científica y Tecnológica (National Agency of Scientific and Technology Promotion of Argentine)"; and especially to "SECyT (Secretary of Science and Technology from the National University of Cordoba (UNC)", Argentina, for awarding us the extension of the Grant for Young Researchers to the author A. G. B. at INFIQC.

Especially thanks to Professor Denis Boudreau from "Département de chimie and Centre d'optique, photonique et laser (COPL)", Québec, Canada, for the long-standing Research Collaboration in progress; as well as to all the Canadian Grants that permit it. And, as well, to Professor Valeria Amé from "Centro de Investigaciones en Bioquímica Clínica e Inmunología (CIBICI), Departamento de Bioquímica Clínica, Facultad de Ciencias Químicas, UNC", Argentine. In the same manner, it should be aknowledge to the co-author Martin Amé, from "Hospital Privado e Instituto Universitario de Ciencias Biomédicas de Córdoba”, Córdoba, Argentine.

\section{References}

1. Ting W (2018) A broadband achromatic metalens for focusing and imaging in the visible. Nature Nanotech 13: 220-226. [Crossref]

2. Huser T (2008) Nano-Biophotonics: new tools for chemical nano-analytics. Current Opinion in Chemical Biology 12: 497-504.

3. Salinas C, Bracamonte AG (2018) Design of Advanced Smart Ultraluminescen Multifunctional Nanoplatforms for Biophotonics and Nanomedicine applications. Frontiers in Drug, Chemistry and Clinical Research 1: 1-8.

4. Zeltins A (2013) Construction and characterization of virus-like particles: A Review. Mol Biotechnol 53: 92-107.

5. Bochner BR (2009) Global phenotypic characterization of bacteria. FEMS Microbiology Reviews 33: 191-205.

6. Lloyd D (1997) Circadian and ultradian clock-controlled rhythms in unicellular microorganisms. Advances in Microbial Physiology 39: 291-338.

7. Lekka M (2012) Cancer cell detection in tissue using AFM. Archives of Biochemistry and Biophysics 518: 151-156.

8. Sengupta S (2020) The story of COVID-19: A comparative analysis. Science Translational Medicine 12: 1-4.

9. Baum A (2020) Antibody cocktail to SARS-CoV-2 spike protein prevents rapid mutational escape seen with individual antibodies Science 10: 1-8.

10. Ellinghaus D (2020) The ABO blood group locus and a chromosome 3 gene cluster associate with SARS-CoV-2 respiratory failure in an Italian-Spanish genome-wide association analysis. MedRxiv 2: 1-23.

11. Botero S (2019) Escherichia coli as a platform for the study of phosphoinositide biology. Sci Adv 5: 1-16.

12. Cavicchioli R, Ripple WJ (2019) Scientists' warning to humanity: microorganisms and climate change. Nature Reviews Microbiology 17: 569-586.

13. Hong X (2020) The pore size of mesoporous silica nanoparticles regulates their antigen delivery efficiency. Sci $A d v$ 6: 1-13.

14. Mishra R (2019) 7-Nanoporous materials, nanotechnology in textiles, theory and application. The Textile Institute Book Series 2: 311-353.

15. Dienerowitz M (2008) Optical manipulation of nanoparticles: A review. Journal of Nanophotonics 2: 1-32.

16. Zhang Q (2019) The post-PAM interaction of RNA-guided spCas9 with DNA dictates its target binding and dissociation. Sci Adv 5: 1-10. 
17. Burns A (2006) Fluorescent core-shell silica nanoparticles: towards "Lab on a Particle" architectures for nanobiotechnology. Chem Soc Rev 35: 1028-1042.

18. Gammoudi I (2013) Characterization of Silica nanoparticles in interaction with escherichia coli bacteria, International Journal of Chemical, Molecular, Nuclear, Materials and Metallurgical Engineering 7: 520-526.

19. Kim D (2020) The Architecture of SARS-CoV-2 Transcriptome. Cell 181: 914-921. [Crossref]

20. Sirohi D, Kuhn RJ (2019) Zika virus structure, maturation, and receptors. The Journal of Infectious Diseases 216: 935-944.

21. Bai N (2011) Characterization of Optical properties of bacterial micro-colonies via the comprehensive morphology analyzer, Proc. SPIE 8027. Sensing for Agriculture and Food Quality and Safety III 5: 1-17.

22. Pohorille A (2009) Self-assembly and function of primitive cell membranes. Research in Microbiology 7: 449-456.

23. Cai Y (2020) Distinct conformational states of SARS-CoV-2 spike protein. Science 10: $1-13$.

24. Rogers TF (2020) Isolation of potent SARS-CoV-2 neutralizing antibodies and protection from disease in a small animal model. Science 20: 1-13

25. Baum A (2020) Antibody cocktail to SARS-CoV-2 spike protein prevents rapid mutational escape seen with individual antibodies. Science 10: 1-8.

26. Wec AZ (2020) Broad neutralization of SARS-related viruses by human monoclonal antibodies. Science 2: 1-12.

27. Singh L (2017) Parboosing, the role of nanotechnology in the treatment of viral infections, Ther Adv Infect Dis 4: 105-131.

28. Wang N (2020) Subunit Vaccines against emerging Pathogenic Human Coronaviruses. Front Microbiol 11: 1-19.

29. Morse JS (2020) Learning from the Past: Possible urgent prevention and treatment options for severe acute, Respiratory Infections Caused by 2019-nCoV. Chem Bio Chem 21: 30-738

30. Szunerits S, Barras A, Khanal M, Pagneux Q (2015) Nanostructures for the inhibition of viral infections, Molecules 20: 14051-14081.

31. Speshock JL, Murdock RC, Braydich-Stolle LK, Schrand AM, Hussain SM (2010) Interaction of silver nanoparticles with Tacaribe virus. Journal of Nanobiotechnology 19: $1-9$.

32. Barras A, Pagneux Q, Sane F (2016) High efficiency of functional carbon nanodots as entry inhibitors of herpes simplex virus type 1. ACS Appl Mater Interfaces 8: $9004-$ 9013.

33. Cagno V, Andreozzi P (2017) Broad-spectrum non-toxic antiviral nanoparticles with a virucidal inhibition mechanism. Nature Materials 2: 1-10.

34. Meléndez-Villanueva MA, Morán-Santibañez K (2019) Virucidal activity of gold nanoparticles synthesized by green chemistry using garlic extract. Viruses 11: 1-13.

35. Chompoosor A, Saha K (2010) The role of surface functionality on acute cytotoxicity, ROS Generation and DNA Damage by Cationic Gold Nanoparticles. Small 6: 22462249.

36. Sondi I, Salopek-Sondi B (2004) Silver nanoparticles as antimicrobial agent: a case study on E. coli as a model for Gram-negative bacteria. Journal of Colloid and Interface Science 275: 177-182.

37. Bracamonte AG (2020) New matter properties and applications based on hybrid graphene-based metamaterials, special issue: design and synthesis of graphene based metamaterials, current graphene science. Bentham Sci Pub 4: 1-4.

38. Salinas C, Bracamonte G (2018) Design of advanced smart ultraluminescent multifunctional nanoplatforms for biophotonics and nanomedicine applications. Frontiers in Drug, Chemistry and Clinical Research 1: 1-8.

39. Yazdi S, Daniel JR, Large N (2016) Reversible shape and plasmon tuning in hollow agau nanorods. Nano Lett 16: 6939-6945.

40. Aslan K, Wu M (2007) Fluorescent core-shell Ag@SiO2 nanocomposites for metalenhanced fluorescence and single nanoparticle sensing platforms. J Am Chem Soc 6 : 1524-1525. [Crossref]

41. Lessard-Viger M (2008) Boudreau, FRET enhancement in core shell Nanoparticles. Nanolett 8: 3066-30718.
42. Rioux M (2017) Synthesis of Ultraluminiscent gold core-shell Nanoparticles as NanoImaging platforms for biosensing applications based on Metal enhanced fluorescence. $R S C A d v 7$ : 10252-10258.

43. Liu C (2020) Super-resolution nanoscopy by coherent control on nanoparticle emission. Sci Adv 6: 1-8.

44. Zhao T (2019) Nanoscopy by coherent control on nanoparticle emission J of Materials Chem 7: 14693-14700.

45. Hong S (2020) Vascular disrupting agent induced aggregation of gold nanoparticles for photothermally enhanced tumor vascular disruption. Sci Adv 6: 1-12.

46. Liua R, Hana H, Liu F, Lv KZ (2020) Positive rate of RT-PCR detection of SARSCoV-2 infection in 4880 cases from one hospital in Wuhan, China, from Jan to Feb 2020. Clinica Chimica Acta 505: 172-175.

47. Fuk-Woo Chan J (2020) Improved molecular diagnosis of COVID-19 by the novel, highly sensitive and specific COVID-19-RdRp/Hel Real-Time reverse TranscriptionPCR assay validated In Vitro and with clinical specimens, $J$ of Clinical Biology 4: 1-10.

48. van Gils MJ (2020) Potent neutralizing antibodies from COVID-19 patients define multiple targets of vulnerability. Science 5: 1-14.

49. Liua R (2020) Positive rate of RT-PCR detection of SARS-CoV-2 infection in 4880 cases from one hospital in Wuhan, China, from Jan to Feb 2020. Clinica Chimica Acta 5: $172-175$.

50. Turano A, Pirali F (1988) Quantification methods in microbiology, laboratory diagnosis of infectious diseases, SpringerVerlag New York 5: 8-13.

51. Drew1 DA (2020) COPE Consortium, rapid implementation of mobile technology for real-time epidemiology of COVID-19. Science 368: 1-10.

52. Holly J. Butler (2019) Shining a light on clinical spectroscopy: Translation of diagnostic IR, 2D-IR and Raman spectroscopy towards the clinic. Clinical Spectroscopy 1: 1-44.

53. Roy S (2019) Spectroscopy goes viral: Diagnosis of hepatitis B and C virus infection from human sera using ATR-FTIR spectroscopy. Clinical Spectroscopy 1: 1-14.

54. Łoczechin A, Séron K (2019) Functional carbon quantum dots as medical countermeasures to human coronavirus. ACS Appl Mater Interfaces 11: 42964-42974.

55. Zhong R, Wang R (2020) Polydopamine doped virus like structured nanoparticles for photoacoustic imaging guided synergistic chemo-/Photothermal therapy. RSC Adv 10: 18016-18024.

56. Ignatovich FV (2006) Optical detection of single nanoparticles and viruses. IEEE Journal of Selected Topics in Quantum Electronics 12: 1-10.

57. Kurdekar AD (2018) Streptavidin conjugated gold nanoclusters as ultrasensitive fluorescent sensor for early diagnosis of HIV infection. Sci Adv 4: 1-10.

58. Zhang Q (2020) Cellular Nanosponges Inhibit SARS-CoV-2 Infectivity. Nano Lett 20 5570-5574.

59. Schmidt H (2016) Single-virus analysis through chip-based optical detection Bioanalysis 8: 867-870.

60. Qiu G (2020) Dual-Functional plasmonic photothermal biosensors for highly accurate severe acute respiratory syndrome coronavirus 2 detection. ACS NANO14: 5268-5277.

61. Brouard MD (2011) Label-free biosensing based on multilayer fluorescen nanocomposites and a cationic polymeric transducer. ACS Nano 5: 1888-1896. [Crossref]

62. Brouard D (2013) Direct molecular detection of SRY gene from unamplified genomic DNA by metal enhanced fluorescence and FRET. Analytical Methods 5: 6896-6899.

63. Brouard B (2014) PCR free blood group genotyping using a nanobiosensor. Vox Sanguinis 2: 1-9.

64. Righini M (2009) Nano-optical trapping of rayleigh particles and escherichia coli bacteria with resonant optical antennas. Nano Lett 9: 3387-3391.

65. Gontero D (2018) Ultraluminescent gold Core@shell nanoparticles applied to individual bacterial detection based on Metal-Enhanced Fluorescence Nanoimaging. J of Nanophotonics 12: 1-12.

66. Gontero D (2020) In flow metal enhanced fluorescence for biolabelling and biodetection. Photochemical \& Photobiological Science. 
67. Salinas C (2020) Synthetic non-classical luminescence generation by enhanced silica nanophotonics based on Nano-Bio-FRET. RSC Advances 10: 20620-20637.

68. Meech S (2011) Biophotonics, Living lasers. Nature Photonics 5: 387-388.

69. Hales JE (2019) Virus lasers for biological detection. Nature Communicaion 10: 1-11.
70. Morse JS (2020) Learning from the Past: Possible urgent prevention and treatment options for severe acute, Respiratory infections caused by 2019-nCoV. ChemBioChem 21: $30-738$

71. Ali ST (2020) Serial interval of SARS-CoV-2 was shortened over time by nonpharmaceutical interventions. Science 4: 1-8.

Copyright: $\mathbb{C} 2020$ Amé M. This is an open-access article distributed under the terms of the Creative Commons Attribution License, which permits unrestricted use, distribution, and reproduction in any medium, provided the original author and source are credited. 